\title{
Genotypic resistance profiles in Chinese patients with chronic hepatitis $B$ virus infection and the efficacy of nucleoside analog rescue therapy
}

This article was published in the following Dove Press journal:

Therapeutics and Clinical Risk Management

12 March 2015

Number of times this article has been viewed

\author{
Ling-Bo Liang \\ Lan-Lan Chen \\ En-Qiang Chen \\ Juan Liao \\ Hong Tang
}

Center of Infectious Disease, State Key Laboratory of Biotherapy, West China Hospital, West China School of Medicine, Sichuan University, Chengdu, People's Republic of China

Correspondence: Hong Tang

Center of Infectious Disease, State Key Laboratory of Biotherapy, West China Hospital, West China School of Medicine, Sichuan University, 37\# Guoxue Lane, Chengdu 61004I, People's Republic of China

Tel +862885423052

Fax +8628 85423052

Email htang6198@hotmail.com
Background and aims: The aims of this study were to explore the correlation between chronic hepatitis B virus (HBV) drug-resistant mutation profiles and the efficacy of nucleoside analog rescue therapy in patients with initial antiviral treatment failure.

Patients and methods: Patients with initial antiviral therapy failure were recruited between January 2011 and January 2013 from the Division of Infectious Disease, West China Hospital, Sichuan University, Chengdu, People's Republic of China. Following drug-resistant mutation testing, eligible patients received nucleoside analog rescue therapy for 24 weeks. The primary endpoint was rescue therapy efficacy, and the secondary endpoint was adverse events.

Results: We recruited 168 patients with chronic HBV infection who had initial antiviral treatment failure. Eighty-nine patients (52.98\%) experienced virological breakthrough (group A); 79 patients (47.02\%) had partial/null response (group B). Among the patients, 102 (102/168, $60.7 \%$ ) carried at least one HBV drug resistance mutation. The prevalence of genotypic resistance was significantly higher in group A than in group B $(P<0.001)$. In addition, 118 patients $(118 / 168,70.2 \%)$ achieved undetectable serum HBV DNA with the nucleoside analog rescue therapy. Rescue therapy $(P=0.002)$ and no evidence of genotypic resistance $(P=0.001)$ were related to a higher rate of virological response.

Conclusion: These data indicate that patients with chronic HBV infection who have initial antiviral therapy failure with or without signs of genotypic resistance may still stand a chance of gaining therapeutic benefit with nucleoside analog rescue therapy.

Keywords: drug-resistance mutation, virological response, nucleoside and nucleotide analogs

\section{Introduction}

Hepatitis B virus (HBV) chronic infection affects about 350 million people worldwide and can result in cirrhosis and hepatocellular carcinoma. ${ }^{1,2}$ Nucleoside analogs such as lamivudine (LAM), adefovir (ADV), entecavir (ETV), and telbivudine (LDT) have potential therapeutic effects against chronic HBV infection. They can markedly suppress HBV replication by suppressing HBV polymerase activity, leading to improved liver function and reduced incidence of fibrosis, cirrhosis, and hepatocellular carcinoma. However, long-term nucleoside analog therapy is associated with the development of antiviral resistance. ${ }^{1,2}$ For example, about $70 \%-80 \%$ of patients develop LAM resistance after 5 -year treatment; ${ }^{2-4} 42 \%$ of hepatitis Be antigen (HBeAg)-positive patients and $29 \%$ of HBeAg-negative patients develop ADV resistance after 5-year treatment; 5,6 and $25 \%$ of $\mathrm{HBeAg}$-positive patients and $11 \%$ of $\mathrm{HBeAg}$-negative patients develop LDT resistance after 2-year treatment. ${ }^{2}$ 
Currently, drug resistance mutation prevalence and types are debatable and complex. In patients with nucleoside analog treatment failure, virological breakthrough is often the first clinical manifestation thereof. ${ }^{5-10}$ Several Phase III clinical trials of nucleoside analogs in treatment-naïve patients revealed that genotypic resistance could be detected in $0 \%-87.5 \%$ of patients experiencing virological breakthrough,,${ }^{6-10}$ and the discrepancy between the rates of virological breakthrough and the rates of genotypic resistance are not fully understood. As reinforcement of adherence in patients with no genotypic resistance can restore virological response, while patients with genotypic resistance require rescue therapy, differentiating between virological breakthrough due to nonadherence and virological breakthrough due to genotypic resistance is very important. Moreover, partial virological response, another form of nucleoside analog treatment failure, is also quite common in both clinical trials and practice. ${ }^{11-17}$ Notably, genotypic resistance also can be observed in more than half of partial/null responders. ${ }^{18}$

Thus, analysis of the emergence of drug resistance mutations in patients with chronic HBV infection treated with nucleoside analogs with virological breakthrough or partial/null response is critical for clinical management, and efficacious rescue therapy is also necessary. Therefore, the objectives of this study were: 1) to evaluate the profiles of drug-resistant mutations in patients with nucleoside analog treatment failure; and 2) to identify the efficacy of rescue therapies and the factors associated with successful virological response.

\section{Patients and methods}

\section{Study design and eligibility of patients}

In this retrospective study, eligible consecutive patients with chronic HBV infection who had been tested for genotypic resistance due to antiviral treatment failure were recruited from the Division of Infectious Disease, West China Hospital, Sichuan University, Chengdu, People's Republic of China between January 2011 and January 2013. All recruited patients were examined by clinicians and were enrolled into the study. The inclusion criteria were as follows: 1) age 18-65 years; 2) presence of hepatitis B surface antigen in the serum for at least 6 months; 3) classified as partial/null responder (defined as failure to achieve undetectable serum HBV DNA with continuous 24-week nucleoside analog therapy) ${ }^{19}$ and virological breakthrough (defined as having HBV DNA increase by $>1$ $\log$ from nadir level or redetection of HBV DNA after having an undetectable result) ${ }^{20}$ according to the types of virological response to previous nucleoside analog treatment, which included LAM, ADV, ETV, and LDT; and 4) tested for HBV drug-resistant mutations and HBV genotypes.

The exclusion criteria were: 1) hepatitis A, hepatitis C, hepatitis $\mathrm{D}$, hepatitis $\mathrm{E}$, or human immunodeficiency virus infection; 2) jaundice caused by obstructive or hemolytic diseases; 3 ) prolonged prothrombin time induced by blood system diseases; 4) drug hepatitis, Wilson disease, alcoholic liver disease, and autoimmune hepatitis; and 5) comorbiditiess and uncontrolled metabolic condition or psychiatric condition. The study protocol was in accordance with the ethical guidelines of the Declaration of Helsinki and was approved by the Ethics Committee of West China Hospital of Sichuan University. Written informed consent was obtained from all participants.

\section{Laboratory tests}

The laboratory data of all patients were reviewed retrospectively and included 1) biochemical tests reflecting hepatocytic damage: serum alanine transaminase (ALT), aspartate transaminase (AST), albumin (ALB), and total bilirubin, all assayed by colorimetric method (MODULAR EVO; Hoffmann-La Roche Ltd, Basel, Switzerland); 2) international standardized ratio for prothrombin time, determined according to the manufacturer's instructions (CA-7000 System; Sysmex, Kobe, Japan); 3) HBV markers: HBV antigens (HBeAg) and antibodies (HBeAb), detected by commercially available enzyme immunoassays (Alisei Quality System; RADIM, Rome, Italy); and 4) HBV DNA, determined by fluorescent quantifying polymerase chain reaction with a low detection limit of $10^{3}$ copies $/ \mathrm{mL}$ (LightCycler 480; Hoffmann-La Roche Ltd).

\section{Mutation analysis}

An HBV gene fragment (nucleotide 54-1,278) encompassing the complete reverse transcriptase $(R T)$ gene was amplified by polymerase chain reaction. Sequencing was performed using an ABI 3500 DNA Analyzer (Hitachi Ltd, Tokyo, Japan). Sequencing data were analyzed using the Vector NTI Suite software package (Thermo Fisher Scientific, Waltham, MA, USA). Mutations at 16 locations (rt173, rt180, rt181, rt184, rt202, rt204, rt207, rt213, rt214, rt215, rt216, rt234, $\mathrm{rt} 236, \mathrm{rt} 237, \mathrm{rt} 238, \mathrm{rt} 250)$ in the $R T$ gene were analyzed. The rt204V/I mutation was defined as a LAM resistance mutation, which also encompassed LDT resistance mutations. The rtA181V/T and rtN236T mutations were defined as ADV resistance mutations. The rtT184A/G/I/S, rtS202G/I, and rtM250L/V mutations, concomitant with the rtM204V/I mutation, were defined as ETV resistance mutations. 


\section{Treatment schedule and follow-up}

Following genotypic resistance testing, patients continued to receive the initial nucleoside analog treatment (LAM, ADV, ETV, or LDT) if they did not carry HBV drug resistance mutations. Otherwise, the clinicians would recommend rescue antiviral therapy involving ADV combined with ETV, LDT, or LAM to patients harboring HBV drug resistance mutations. All patients were given nucleoside analog treatment for 24 weeks and were observed every week for the first 3 months and every 1 month thereafter until July 2013. The clinical and laboratory data, adverse events, and compliance of all patients were monitored during follow-up.

\section{Statistical analysis}

Quantitative data are expressed as mean \pm standard deviation or median and range, and $t$-test analysis was used. A chisquare test or Fisher's exact test were performed to calculate differences in qualitative data. Independent factors associated with the rate of virological response to rescue therapy were assessed using the logistic regression model. A $P$-value of less than 0.05 was considered to indicate statistical significance. Data were analyzed using the statistical software Intercooled Stata version 8.2 for Windows (StataCorp LP, College Station, TX, USA).

\section{Results}

\section{Demographic data and baseline characteristics}

A total of 168 patients with chronic HBV infection who had initial antiviral therapy failure were recruited. Eighty-nine patients $(52.98 \%)$ experienced virological breakthrough (group A) and 79 patients (47.02\%) had partial/null response (group B). The characteristics of all patients are summarized in Table 1. Except for age, $\mathrm{HBeAg}(+/-)$, and $\mathrm{HBeAb}$ $(+/-)$, there were no significant differences in the baseline characteristics sex, serum ALT, AST, ALB, total bilirubin, international standardized ratio, HBV DNA level, as well as HBV genotype between the two groups.

\section{Genotypic resistance mutations and virological response types}

Among the eligible patients, $102(60.7 \%)$ carried at least one HBV drug resistance mutation, and the remaining $66(39.3 \%)$ harbored wild-type HBV. In particular, the majority of patients $(72 / 102,70.6 \%)$ with drug resistance mutations experienced virological breakthrough, and the majority of patients $(49 / 66,74.2 \%)$ with wild-type HBV had partial/null response. At the same time, the prevalence of genotypic resistance was significantly higher in group A $(72 / 89,80.9 \%)$, of which more experienced virological breakthrough than did patients in group B $(30 / 79,38.0 \%$; $P<0.001)$.

\section{Genotypic resistance, virological response, and initial antiviral therapy}

The relationship among the virological response types, prevalence of genotypic resistance, and initial antiviral treatment are summarized in Table 2. Among all eligible patients, $101(61.1 \%)$ received nucleoside analog monotherapy, $34(20.2 \%)$ received sequential nucleoside analog therapy, and 33 (19.6\%) received nucleoside analog monotherapy and combined therapy prior to genotypic resistance testing. The rate of genotypic resistance was significantly different

Table I Demographic data and baseline characteristics

\begin{tabular}{|c|c|c|c|c|}
\hline & Group A $(n=89)$ & Group B (n=79) & $\chi^{2} / F$ & $P$-values \\
\hline Age (years) ${ }^{\mathrm{a}}$ & $37.61 \pm 11.04$ & $35.58 \pm 8.57$ & 4.51 & 0.06 \\
\hline Sex (male/female) & $63 / 26$ & $62 / 17$ & 1.30 & 0.25 \\
\hline $\operatorname{ALT}(I U / L)^{b}$ & |35.49 (|8-|,| |40) & $117.06(17-952)$ & 0.39 & 0.53 \\
\hline AST (IU/L) ${ }^{b}$ & $68.59(10-544)$ & $65.26(12-452)$ & 0.06 & 0.81 \\
\hline $\operatorname{ALB}(g / L)^{a}$ & $45.33 \pm 5.51$ & $43.91 \pm 6.21$ & 0.71 & $0.4 \mathrm{I}$ \\
\hline TBIL $(\mathrm{mg} / \mathrm{dL})^{\mathrm{a}}$ & $18.95 \pm 9.84$ & $18.62 \pm 10.67$ & 0.01 & 0.91 \\
\hline INR for $\mathrm{PT}^{\mathrm{a}}$ & $1.27 \pm 0.55$ & $1.21 \pm 1.03$ & 1.54 & 0.22 \\
\hline $\mathrm{HBeAg}(+/-)$ & $58 / 31$ & $63 / 16$ & 4.41 & 0.04 \\
\hline $\mathrm{HBeAb}(+/-)$ & $27 / 62$ & $11 / 68$ & 6.44 & 0.01 \\
\hline HBV DNA level (log 10 copies $/ \mathrm{mL})^{\mathrm{a}}$ & $6.12 \pm 2.02$ & $5.82 \pm 1.87$ & 2.38 & 0.15 \\
\hline HBV genotype B/C & $61 / 28$ & $45 / 34$ & 2.41 & 0.12 \\
\hline
\end{tabular}

Notes: ${ }^{\mathrm{a} D a t a}$ are presented as the mean \pm standard deviation. ${ }^{b}$ Data are presented as the median and range. Group A patients experienced virological breakthrough; group $B$ patients had partial/null response.

Abbreviations: ALB, albumin; ALT, alanine transaminase; AST, aspartate transaminase; $\mathrm{HBeAb}$, hepatitis Be antibodies; $\mathrm{HBeAg}$, hepatitis Be antigen; $\mathrm{HBV}$, hepatitis B virus; INR, international standard ratio; PT, prothrombin time; TBIL, total bilirubin. 
Table 2 Genotypic resistance and virological response in relation to previous antiviral therapy

\begin{tabular}{|c|c|c|c|}
\hline $\begin{array}{l}\text { Previous antiviral therapy } \\
\text { patterns }(n=168)\end{array}$ & $\begin{array}{l}\text { Virological breakthrough } \\
(n=89)\end{array}$ & $\begin{array}{l}\text { Partial/null response } \\
(n=79)\end{array}$ & $\begin{array}{l}\text { Genotypic resistance } \\
(n=102)\end{array}$ \\
\hline Monotherapy $(n=|0|)$ & 70 (70/10।, 69.3\%) & $3 \mathrm{I}(3 \mathrm{I} / \mathrm{IOI}, 30.7 \%)$ & 58 (58/I0I, 57.4\%) \\
\hline LAM $(n=47)$ & $43(43 / 47,91.5 \%)$ & $4(4 / 47,8.5 \%)$ & $37(37 / 47,78.7 \%)$ \\
\hline $\operatorname{ADV}(n=36)$ & $14(14 / 36,38.9 \%)$ & $22(22 / 36,61.1 \%)$ & $10(10 / 36,27.8 \%)$ \\
\hline LDT $(n=17)$ & $13(13 / 17,76.5 \%)$ & $4(4 / 17,23.5 \%)$ & II (II/I7, 64.7\%) \\
\hline $\operatorname{ETV}(n=I)$ & 0 & 1 & 0 \\
\hline Sequential therapy $(n=34)$ & $17(17 / 34,50 \%)$ & $17(17 / 34,50 \%)$ & $28(28 / 34,82.4 \%)$ \\
\hline$A D V \rightarrow$ LAM $(n=14)$ & $4(4 / 14,28.6 \%)$ & $10(10 / 14,71.4 \%)$ & $9(9 / 14,64.3 \%)$ \\
\hline $\mathrm{ADV} \rightarrow \mathrm{LDT}(\mathrm{n}=3)$ & 0 & 3 & $3(3 / 3,100 \%)$ \\
\hline $\mathrm{LAM} \rightarrow \mathrm{ETV}(\mathrm{n}=17)$ & $13(13 / 17,76.5 \%)$ & $4(4 / 17,23.5 \%)$ & $16(16 / 17,94.1 \%)$ \\
\hline Monotherapy to combined therapy $(n=33)$ & $2(2 / 33,6.1 \%)$ & 31 (3I/33, 93.9\%) & $16(16 / 33,48.5 \%)$ \\
\hline LAM $\rightarrow$ LAM + ADV $(n=3)$ & 0 & 3 & I (I/3, 33.3\%) \\
\hline$A D V \rightarrow L A M+A D V(n=28)$ & $2(2 / 28,7.1 \%)$ & $26(26 / 28,92.9 \%)$ & $13(13 / 28,46.4 \%)$ \\
\hline $\mathrm{LDT} \rightarrow \mathrm{LDT}+\mathrm{ADV}(\mathrm{n}=2)$ & 0 & 2 & $2(2 / 2,100 \%)$ \\
\hline
\end{tabular}

Abbreviations: ADV, adefovir; ETV, entecavir; LAM, lamivudine; LDT, telbivudine.

in 58 patients $(58 / 101,57.4 \%)$ treated with monotherapy, in 28 patients $(28 / 34,82.4 \%)$ treated with sequential therapy, and in 16 patients $(16 / 33,48.5 \%)$ treated with monotherapy and combined therapy $(P=0.010)$.

Specifically, compared with patients who received ADV monotherapy, patients who received LAM or LDT monotherapy had significant virological breakthrough and genotypic resistance rate $(P<0.001)$. There was also a significant difference in genotypic resistance and virological response between ADV to LAM and LAM to ETV sequential therapy $(P=0.018)$. In addition, 28 patients $(28 / 33,84.8 \%)$ received ADV monotherapy followed by ADV and LAM combined therapy, and almost all $(31 / 33,93.9 \%)$ were partial/null responders.

\section{Genotypic resistance and HBV genotypes}

The genotypic resistance and HBV genotypes are listed in Table 3. There was a slight trend toward more drug resistance mutations in patients with HBV genotype B (65/106, 61.3\%) over patients with HBV genotype C (37/62, 59.7\%), although this difference was not statistically significant $(P=0.833)$. Compared to patients with HBV genotype B, significantly more patients with HBV genotype $\mathrm{C}$ had rtA181T/V mutations $(P=0.02)$. However, the M204V/I, N236T, T184A/I/S, and S202G mutations in patients with HBV genotypes were not significantly different (all $P>0.05$ ).

\section{Virological response types, genotypic resistance mutation, and rescue antiviral therapy}

Seven patients $(7 / 168,4.17 \%)$ continued to receive the initial nucleoside analog treatment (LAM, ADV, ETV, or LDT) because they carried wild-type HBV. Rescue therapy (ADV combined with ETV, LDT, or LAM) was recommended for the remaining 161 patients $(161 / 168$, $95.83 \%)$. Among the patients who received rescue therapy, $88(88 / 161,54.66 \%)$ received ETV + ADV combined therapy, and $73(73 / 161,45.34 \%)$ received LAM/LDT + ADV combined therapy.

Overall, 118 patients $(118 / 168,70.2 \%)$ achieved undetectable serum HBV DNA with rescue therapy after a follow-up of 24 weeks. Compared to the patients with drug-resistant HBV (64/102, 62.7\%), the patients harboring wild-type HBV had significantly higher virological response $(54 / 66$, $81.8 \% ; P=0.008)$, as shown in Figure 1A. In addition, when stratifying patients according to rescue therapy type, we found that the rate of virological response in patients who received $\mathrm{ETV}+\mathrm{ADV}$ rescue therapy was significantly

Table 3 Correlation between drug resistance mutation and hepatitis B virus genotypes

\begin{tabular}{llll}
\hline Mutation location & Genotype B (n=106) & Genotype C (n=62) & P-value \\
\hline M204V/I $(n=77)$ & $53(50 \%)$ & $24(38.7 \%)$ & 0.156 \\
N236T $(n=10)$ & $6(5.7 \%)$ & $4(6.5 \%)$ & 0.834 \\
AI8IT/V $(n=11)$ & $3(2.8 \%)$ & $8(I 2.9 \%)$ & 0.020 \\
TI84A/I/S $(n=7)$ & $6(5.7 \%)$ & $1(1.6 \%)$ & 0.205 \\
S202G $(n=6)$ & $5(4.7 \%)$ & $1(1.6 \%)$ & 0.295 \\
\hline
\end{tabular}

Note: Bold represents a statistically significant value. 

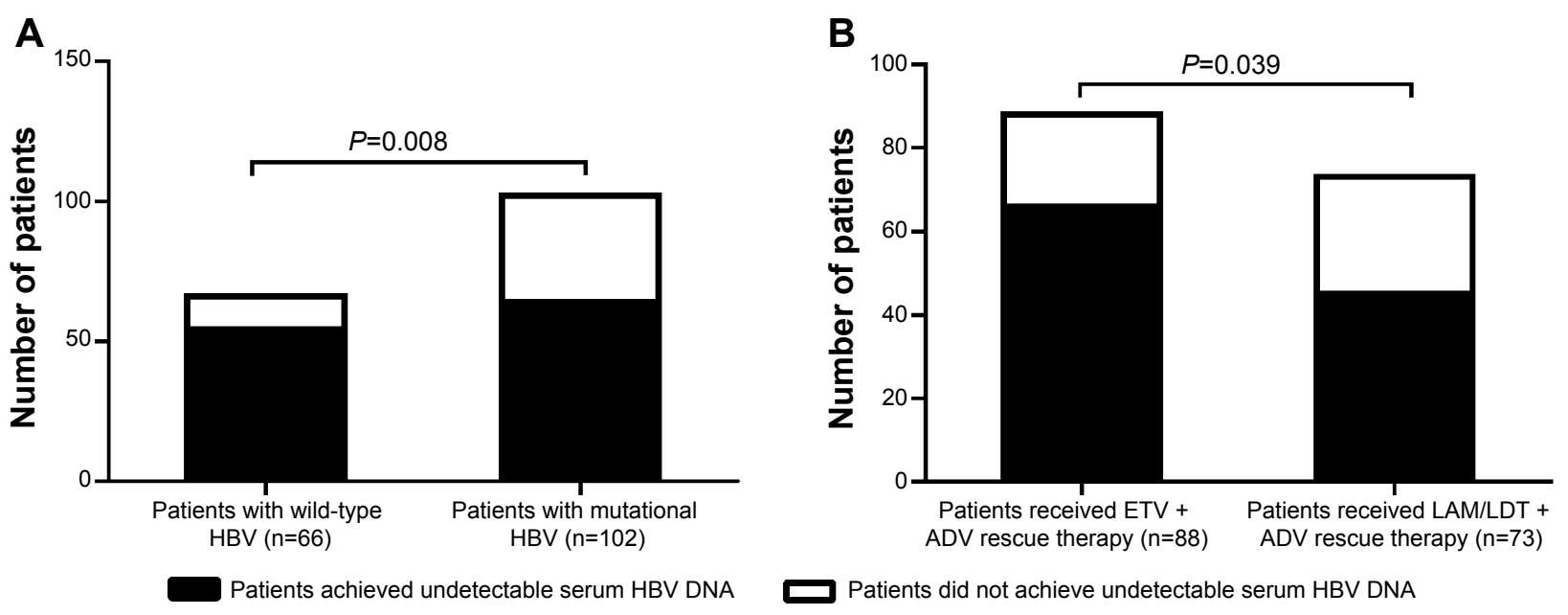

Figure I The comparisons of the rates of virological response in relation to genotypic resistance and rescue therapy pattern.

Notes: (A) Compared to the patients with drug-resistant HBV, the patients harboring wild-type HBV had significantly higher virological response ( $P=0.008)$. (B) The rate of virological response in patients who received ETV + ADV rescue therapy was significantly higher than that in patients who received LAM/LDT + ADV rescue therapy ( $P=0.039)$. Abbreviations: ADV, adefovir; ETV, entecavir; HBV, hepatitis B virus; LAM, lamivudine; LDT, telbivudine.

higher than that in patients who received LAM/LDT + ADV rescue therapy $(P=0.039)$, as shown in Figure $1 \mathrm{~B}$.

\section{Factors associated with rescue therapy efficacy}

Logistic regression analyses demonstrated that rescue therapy $(P=0.002)$ and no evidence of genotypic resistance $(P=0.001)$ were related to a higher rate of virological response. On the contrary, the initial treatment $(P=0.018)$ and partial/null response to initial treatment $(P=0.004)$ were related to a lower rate of virological response.

\section{Safety}

There were no adverse events in patients who received nucleoside analog rescue therapy. Therefore, we concluded that there is no significant correlation between nucleoside analog rescue therapy and adverse events.

\section{Discussion}

The present study findings demonstrate that patients with chronic HBV infection who have initial antiviral therapy failure with or without signs of genotypic resistance may still stand a chance of benefiting therapeutically from nucleoside analog rescue therapy. Importantly, these data provide a rationale for genotypic resistance testing in clinical practice, which would detect partial/null responses in a timely manner so that patients can change their treatment strategy before drug resistance mutations occur.

By stratifying patients according to the initial antiviral therapy, we found that our results were consistent with those of several previous studies, which showed that the main manifestation of treatment failure in patients who received LAM nucleoside analog monotherapy was virological breakthrough accompanied by a high rate of genotypic resistance. ${ }^{12,14}$ However, in patients treated with ADV monotherapy, the main manifestation of treatment failure was partial/null response, and the rate of genotypic resistance was relatively low. ${ }^{7,21}$ It was also noted that there was up to $64.3 \%$ genotypic resistance in ADV-treated patients who experienced virological breakthrough, which was significantly higher than that in partial/null responders. Therefore, once patients treated with ADV monotherapy show signs of inefficacy, clinicians should be aware of the need to perform genotypic resistance testing early, as it appears likely that virological breakthrough may have occurred.

Unsurprisingly, almost all patients who receive sequential nucleoside analog therapy had genotypic resistance. ${ }^{22,23}$ In addition, there was a significant difference in genotypic resistance and virological response between ADV to LAM and LAM to ETV sequential therapy, and these results suggest that nucleoside analog rescue therapy should be administered when patients experience virological breakthrough, as these patients may have developed drug-resistant mutations. The majority of patients who received nucleoside analog monotherapy and combined therapy because of poor virological response to initial antiviral treatment received ADV monotherapy followed by ADV and LAM combined therapy, and almost all of them were partial/null responders.

Our study showed a slight trend toward more drug resistance mutations in patients with $\mathrm{HBV}$ genotype $\mathrm{B}$ over patients with $\mathrm{HBV}$ genotype $\mathrm{C}$, although it was not statistically significant. Our results are consistent with those of several 
previous studies that showed that HBV genotype B is associated with a higher risk of developing mutations, but the reasons remain unknown and warrant further research. ${ }^{24,25}$ Compared to patients with $\mathrm{HBV}$ genotype $\mathrm{B}$, significantly more patients with HBV genotype $\mathrm{C}$ had the rtA181T/V mutation. However, the M204V/I, N236T, T184A/I/S, and S202G mutations in patients with HBV genotypes did not differ significantly.

In our study, most patients accepted rescue combined therapy after genotypic resistance testing; the exception was seven patients without evidence of genotypic resistance, who continued to receive their previous monotherapy. After a 24-week follow-up, the overall rate of virological response was $70.2 \%$. Compared to the patients with drug-resistant $\mathrm{HBV}$, patients harboring wild-type $\mathrm{HBV}$ had significantly higher virological response. Therefore, these results suggest that patients with initial antiviral treatment failure but without evidence of genotypic resistance have a higher chance of achieving therapeutic success compared with patients who have already developed drug resistance.

In addition, when stratifying patients according to rescue therapy type, we noted that the rate of virological response in patients who received ETV + ADV rescue therapy was significantly higher than that of patients who received LAM/ LDT + ADV rescue therapy. Furthermore, multiple regression analyses showed that ETV + ADV rescue therapy was related to a higher rate of virological response. Interestingly, in 66 patients with wild-type HBV, switching to ETV + ADV follow-up therapy was independently correlated with a higher rate of successful virological response. In contrast, exposure to more than one nucleoside analog correlated independently with a lower rate of successful virological response. This indicates that patients with no evidence of genotypic resistance but with initial treatment failure require a salvage treatment "upgrade", as do patients who already have genotypic resistance, especially those who have been exposed to more than one nucleoside analog.

\section{Conclusion}

This study demonstrates that drug-resistant mutations may be the main cause of nucleoside analog treatment failure in patients experiencing virological breakthrough. Once patients treated with initial antiviral treatment show signs of inefficacy, clinicians should perform genotypic resistance testing early because it appears likely that virological breakthrough may have occurred. HBV patients with initial antiviral therapy failure who do not show signs of genotypic resistance may still stand a chance of benefiting therapeutically from nucleoside analog rescue therapy. Importantly, these data provide a rationale for genotypic resistance testing in the clinic, which should detect partial/null responders in a timely manner so that patients can change their treatment strategy before drug resistance mutations occur. Also, these results may be applied to Chinese (Asian) chronic HBV infection patients, and further studies will define the correlation between chronic HBV drug-resistant mutation profiles and the efficacy of nucleoside analog rescue therapy.

\section{Acknowledgment}

This research was supported by the National Twelve-Five Project of China, and no benefits in any form have been or will be received from a commercial party directly or indirectly related to the subject of this article.

\section{Disclosure}

The authors report no conflicts of interest in this work.

\section{References}

1. Pawlotsky JM, Dusheiko G, Hatzakis A, et al. Virologic monitoring of hepatitis B virus therapy in clinical trials and practice: recommendations for a standardized approach. Gastroenterology. 2008;134(2): 405-415.

2. Dienstag JL. Hepatitis B virus infection. N Engl J Med. 2008;359(14): 1486-1500.

3. Zoulim F, Perrillo R. Hepatitis B: reflections on the current approach to antiviral therapy. J Hepatol. 2008;48 Suppl 1:S2-S19.

4. Lai CL, Dienstag J, Schiff E, et al. Prevalence and clinical correlates of YMDD variants during lamivudine therapy for patients with chronic hepatitis B. Clin Infect Dis. 2003;36(6):687-696.

5. Lok AS, Lai CL, Leung N, et al. Long-term safety of lamivudine treatment in patients with chronic hepatitis B. Gastroenterology. 2003; 125(6):1714-1722.

6. Zoulim F, Locarnini S. Hepatitis B virus resistance to nucleos(t)ide analogues. Gastroenterology. 2009;137(5):1593-1608.

7. Hadziyannis SJ, Tassopoulos NC, Heathcote EJ, et al; Adefovir Dipivoxil 438 Study Group. Long-term therapy with adefovir dipivoxil for $\mathrm{HBeAg-negative} \mathrm{chronic} \mathrm{hepatitis} \mathrm{B} \mathrm{for} \mathrm{up} \mathrm{to} 5$ years. Gastroenterology. 2006;131(6):1743-1751.

8. Marcellin P, Chang TT, Lim SG, et al. Long-term efficacy and safety of adefovir dipivoxil for the treatment of hepatitis B e antigen-positive chronic hepatitis B. Hepatology. 2008;48(3):750-758.

9. Liaw YF, Gane E, Leung N, et al; GLOBE Study Group. 2-Year GLOBE trial results: telbivudine is superior to lamivudine in patients with chronic hepatitis B. Gastroenterology. 2009;136(2):486-495.

10. Lok AS, McMahon BJ. Chronic hepatitis B: update 2009. Hepatology. 2009;50(3):661-662.

11. Hadziyannis SJ, Tassopoulos NC, Heathcote EJ, et al; Adefovir Dipivoxil 438 Study Group. Adefovir dipivoxil for the treatment of hepatitis B e antigen-negative chronic hepatitis B. N Engl J Med. 2003;348(9):800-807.

12. Chang TT, Gish RG, de Man R, et al; BEHoLD AI463022 Study Group. A comparison of entecavir and lamivudine for $\mathrm{HBeAg}$-positive chronic hepatitis B. N Engl J Med. 2006;354(10):1001-1010.

13. Lai CL, Shouval D, Lok AS, et al; BEHoLD AI463027 Study Group. Entecavir versus lamivudine for patients with $\mathrm{HBeAg}$-negative chronic hepatitis B. N Engl J Med. 2006;354(10):1011-1020.

14. Lai CL, Gane E, Liaw YF, et al; Globe Study Group. Telbivudine versus lamivudine in patients with chronic hepatitis B. N Engl J Med. 2007; 357(25):2576-2588. 
15. Marcellin P, Heathcote EJ, Buti M, et al. Tenofovir disoproxil fumarate versus adefovir dipivoxil for chronic hepatitis B. N Engl J Med. 2008; 359(23):2442-2455.

16. Chon YE, Park JY, Ahn SH, et al. Partial virological response to adefovir add-on lamivudine rescue therapy in patients with lamivudine-resistant chronic hepatitis B. Digestion. 2013;87(3):196-203.

17. Kwon DH, Kim IH, Choung BS, et al. Continuous long-term entecavir therapy in naïve chronic hepatitis B patients showing partial virologic response. Gut Liver. 2013;7(6):712-718.

18. Salpini R, Alteri C, Cento V, et al. Snapshot on drug-resistance rate and profiles in patients with chronic hepatitis $\mathrm{B}$ receiving nucleos $(\mathrm{t})$ ide analogues in clinical practice. J Med Virol. 2013;85(6):996-1004.

19. European Association For The Study Of The Liver. EASL clinical practice guidelines: management of chronic hepatitis B virus infection. J Hepatol. 2012;57(1):167-185.

20. Hongthanakorn C, Chotiyaputta W, Oberhelman K, et al. Virological breakthrough and resistance in patients with chronic hepatitis $\mathrm{B}$ receiving nucleos(t)ide analogues in clinical practice. Hepatology. 2011;53(6): 1854-1863.
21. Ghany MG, Doo EC. Antiviral resistance and hepatitis B therapy. Hepatology. 2009;49(5 Suppl):S174-S184.

22. Mutimer D, Pillay D, Cook P, et al. Selection of multiresistant hepatitis B virus during sequential nucleoside-analogue therapy. J Infect Dis. 2000;181(2):713-716.

23. Kim SS, Cho SW, Kim SO, Hong SP, Cheong JY. Multidrug-resistant hepatitis B virus resulting from sequential monotherapy with lamivudine, adefovir, and entecavir: clonal evolution during lamivudine plus adefovir therapy. J Med Virol. 2013;85(1):55-64.

24. Liu CJ, Kao JH. Global perspective on the natural history of chronic hepatitis B: role of hepatitis B virus genotypes A to J. Semin Liver Dis. 2013;33(2):97-102.

25. Hsieh TH, Tseng TC, Liu CJ, et al. Hepatitis B virus genotype B has an earlier emergence of lamivudine resistance than genotype C. Antivir Ther. 2009;14(8):1157-1163.
Therapeutics and Clinical Risk Management

\section{Publish your work in this journal}

Therapeutics and Clinical Risk Management is an international, peerreviewed journal of clinical therapeutics and risk management, focusing on concise rapid reporting of clinical studies in all therapeutic areas, outcomes, safety, and programs for the effective, safe, and sustained use of medicines. This journal is indexed on PubMed Central, CAS,

\section{Dovepress}

EMBase, Scopus and the Elsevier Bibliographic databases. The manuscript management system is completely online and includes a very quick and fair peer-review system, which is all easy to use. Visit http://www.dovepress.com/testimonials.php to read real quotes from published authors.

Submit your manuscript here: http://www.dovepress.com/therapeutics-and-clinical-risk-management-journal 